\title{
Time forecast of a break-off event from a hanging glacier
}

\author{
Jérome Faillettaz ${ }^{1}$, Martin Funk ${ }^{2}$, and Marco Vagliasindi ${ }^{3}$ \\ ${ }^{1} 3 \mathrm{G}$, UZH, University of Zürich, Zürich, Switzerland \\ ${ }^{2}$ VAW, ETHZ, Zürich, Switzerland \\ ${ }^{3}$ Fondazione Montagna Sicura, Courmayeur, Aosta Valley, Italy \\ Correspondence to: Jérome Faillettaz (jerome.faillettaz@geo.uzh.ch)
}

Received: 5 August 2015 - Published in The Cryosphere Discuss.: 17 September 2015

Revised: 3 May 2016 - Accepted: 12 May 2016 - Published: 3 June 2016

\begin{abstract}
A cold hanging glacier located on the south face of the Grandes Jorasses (Mont Blanc, Italy) broke off on the 23 and 29 September 2014 with a total estimated ice volume of $105000 \mathrm{~m}^{3}$. Thanks to accurate surface displacement measurements taken up to the final break-off, this event was successfully predicted 10 days in advance, enabling local authorities to take the necessary safety measures. The breakoff event also confirmed that surface displacements experienced a power law acceleration along with superimposed $\log$-periodic oscillations prior to the final rupture. This paper describes the methods used to achieve a satisfactory time forecast in real time and demonstrates, using a retrospective analysis, their potential for the development of early-warning systems in real time.
\end{abstract}

\section{Introduction}

Rockfalls, rock instabilities due to permafrost degradation, landslides, snow avalanches or avalanching glacier instabilities are gravity-driven rupture phenomena occurring in natural heterogeneous media. Such events have a potential to cause major disasters, especially when they are at the origin of a chain of processes involving other materials such as snow (snow avalanche), water (flood) and/or debris (mudflow). The reliable forecasting of such catastrophic phenomena combined with a timely evacuation of the endangered areas is often the most effective way to cope with such natural hazards. Unfortunately, accurate time prediction of such events remains a somewhat daunting task as (i) natural materials are heterogeneous, (ii) the heterogeneity is difficult to quantify and measure and (iii) the rupture is a nonlinear process involving such heterogeneities. Although often lo- cated in a remote high-mountain environment, avalanching glacier instabilities offer an interesting starting point for investigating early-warning perspectives of break-off events, as a glacier consists of a single material (ice) lying on welldefined bedrock. This relative simplicity of the system allows the focus to be placed on the rupture processes leading to the initiation of the instability. Recently, considerable efforts in monitoring, analyzing and modeling such phenomena have led to significant advances in understanding the destabilization process and in improving early-warning perspectives (Faillettaz et al., 2015).

In general, it is possible to distinguish three types of avalanching glacier instabilities according to the thermal properties of their ice/bedrock interface (Faillettaz et al., $2011 \mathrm{~b}, 2012,2015)$. If temperate, the presence of liquid water in the glacier plays a key role in the initiation and the development of the instability as its presence influences the basal properties of the ice/bedrock interface (diminution of friction, lubrication or loss of support). In such cases, several preliminary conditions to be fulfilled can be identified, but an accurate time forecast of an impending break-off event is still far from being possible. If the ice/bed interface experiences a transition from cold to temperate, the presence of meltwater may reduce the basal resistance, which promotes the instability. No clear and easily detectable precursory signs are known in this case, and the only way to infer any potential instability is to monitor the temporal evolution of the thermal regime. If the ice/bedrock is cold, glaciers are entirely frozen to their bedrock. This situation appears in the case of high-altitude hanging glaciers located entirely in accumulation zones. The snow accumulation is mostly compensated by the periodic break-off of ice chunks (Pralong and Funk, 2006), occurring once a critical point in glacier geometry is 
reached. The instability results from the progressive accumulation of internal damage due to an increasing stress regime caused by glacier thickening. In this case, the rupture occurs within the ice, immediately above the bedrock (see Fig. 12d in Pralong and Funk, 2006). The maturation of the rupture was shown to be associated with a typical time evolution of both surface velocities (Faillettaz et al., 2008) and passive seismic activity (Faillettaz et al., 2011a). This characteristic time evolution can theoretically be used to predict the occurrence of a catastrophic event. This was done a posteriori with data obtained prior to the 2005 break-off of the Weisshorn glacier.

In this context, the Whymper glacier, a cold hanging glacier located at the Grandes Jorasses (Mont Blanc, Alps, Italy), had already broken off several times in the past, leading to major ice avalanches that occasionally reached the bottom of the valley. In autumn 2008, the glacier recovered its previous critical geometry from the year 1998 and again a critical crevasse appeared approximatively $100 \mathrm{~m}$ upstream the frontal cliff, prompting the local authorities to initiate a monitoring program to enable a time forecast of the expected break-off event. The glacier finally broke off causing no damage in autumn 2014, after more than 5 years of monitoring. The break-off event was successfully predicted 2 weeks in advance.

The aim of this paper is to confirm the validity of the time forecast procedure first developed in 2005 on the Weisshorn glacier based on a unique data set of surface displacements up to the final break-off event. In all previous studies these records stopped several days prior to the failure.

After describing the glacier and the monitoring system installed on the glacier, we analyze the time evolution of the surface displacement measurements in the context of a time forecast procedure. While comparing this break-off event with the Weisshorn event of 2005 we discuss the results obtained, with the goal of improving the understanding of this phenomenon.

\section{Grandes Jorasses glacier}

\subsection{Study site}

The Whymper glacier is located on the south face of the Grandes Jorasses (Mont Blanc massif, Italy) between 3900 and $4200 \mathrm{~m}$ a.s.l. (Fig. 1). The front of the glacier is about $90 \mathrm{~m}$ wide and its surface area amounts to $25000 \mathrm{~m}^{2}$. This very steep cold hanging glacier (about $40^{\circ}$ ) lies above the village of Planpincieux and the Italian Val Ferret, a famous and highly frequented tourist destination both in winter and summer. In 1997, six boreholes were drilled down to the bed and temperature profiles were measured, indicating basal temperatures below the freezing point (below $-1.6 \pm 0.4{ }^{\circ} \mathrm{C}$ ) at all locations (Pralong and Funk, 2006). Historical data and morphological evidence indicate that the glacier experienced re-

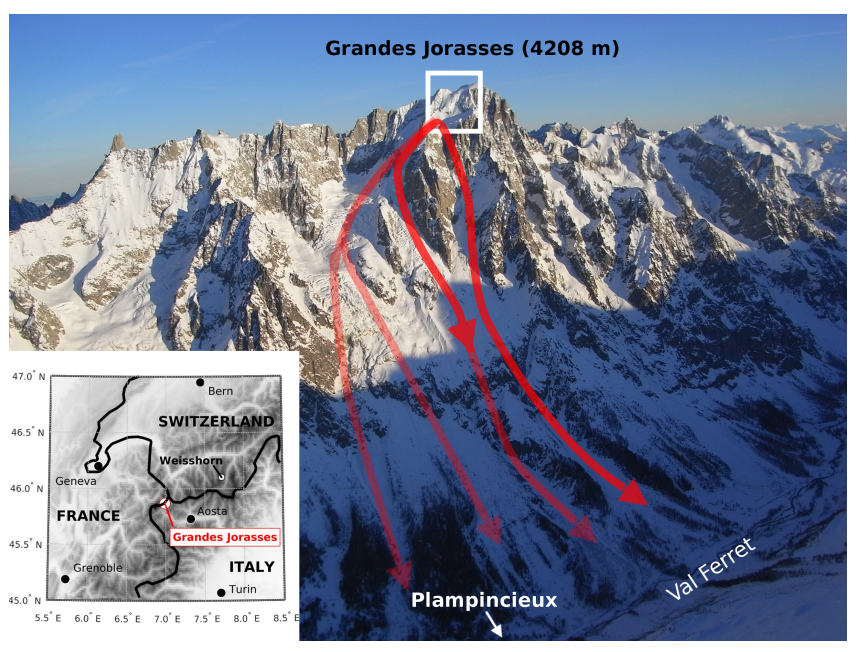

Figure 1. Global view of the Val Ferret with Grandes Jorasses. The white rectangle highlights Whymper glacier. The light red lines indicate possible avalanche flow path (for more details see Margreth et al., 2011); red lines indicate avalanche path from the 1998 breakoff event. The inset shows geographical situation of the studied glacier.

current break-off events that can be dangerous, particularly in winter, when the initial ice avalanche can drag snow in its path. This hanging glacier had periodically broken off in the past, leading to large avalanches that reached the bottom of the valley.

\subsection{Break-off event history}

The glacier has broken off several times during the last 100 years. Some of these events have been observed and reported.

- On 21 December 1952, after an intensive snowfall period, a huge avalanche was released below the Grandes Jorasses which destroyed a 200-year old forest and blocked the bottom of the Val Ferret over a distance of more than $1 \mathrm{~km}$. The avalanche volume was estimated at more than $1000000 \mathrm{~m}^{3}$. It is not clear whether the snow avalanche was triggered by an ice avalanche from the Whymper glacier.

- In August 1993 and July 1996, the glacier released ice avalanches of 80000 and $24000 \mathrm{~m}^{3}$, respectively. These ice avalanches did not reach the bottom of the valley.

- The last major break-off event occurred in the night of 31 May to 1 June 1998. Almost the entire Whymper glacier (around $150000 \mathrm{~m}^{3}$ ) broke off at one time and the triggered ice avalanche reached the bottom of the valley, fortunately without causing damage (Fig. 1). According to Pralong and Funk (2006), the formation of the upper crevasse was observed 2.5 years before failure. 

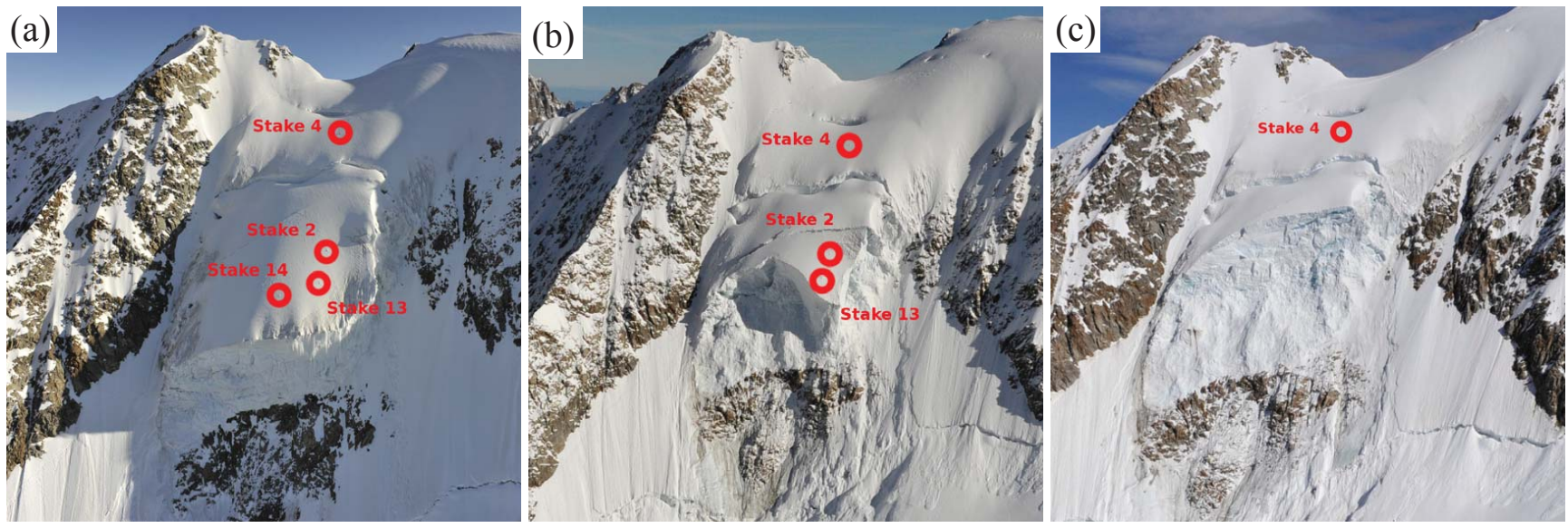

Figure 2. (a) Grandes Jorasses (Whymper) glacier before (23 August 2014), (b) after the first break-off (23 September 2014) and (c) after the second break-off (30 September 2014).

\subsection{Present monitoring: 2009-2014}

The survey primarily consisted of surface displacement measurements with an automatic total station and GPS as well as close-range photogrammetry (Margreth et al., 2011). Two reflectors set on the rock on both sides of the glacier were used as reference, and several reflectors mounted on stakes were directly drilled into the ice, so that their exact positions could be monitored (Fig. 2). Because of instrument problems, the seismic activity unfortunately could not be monitored as initially planned.

Starting in 2010, surface displacements were continuously recorded at several stakes at $2 \mathrm{~h}$ intervals (when the prisms were visible, i.e., good weather conditions) with the aim to detect an impending ice avalanche in a timely manner (Margreth et al., 2011). Using the same correction technique as described by Faillettaz et al. (2008) (Sect. 4.1), the surface displacements could be determined with an accuracy better than $1 \mathrm{~cm}$, allowing surface velocities to be inferred.

In parallel to the monitoring program, a safety concept for the valley floor was developed, considering several scenarios of falling ice volumes. The different ice avalanche scenarios were simulated using the two-dimensional calculation model RAMMS (Christen et al., 2010). The necessary safety measures were defined according to the local avalanche danger level and the potential volume of a break-off event (Margreth et al., 2011).

\subsection{The 2014 break-off event}

From 2010 on, surface displacements were surveyed without interruption. The Whymper glacier finally broke off, with an estimated ice volume similar to the 1998 event (about $105000 \mathrm{~m}^{3}$ ). Contrary to the 1998 event, the glacier broke off in two events on 23 and 29 September 2014, without reaching the valley (Fig. 2). At the final break-off, four reflectors were still active, two of them having been in place for more than 2 years. Despite poor weather conditions between 16 and 21 September, the monitoring was operational up to the final break-off. By chance, there was one reflector on each of the two unstable parts and one on the stable part (Fig. 2).

Striking qualitative analogies with those of the 2005 Weisshorn event (Faillettaz et al., 2008) can be highlighted.

1. This steep cold hanging glacier experiences periodic break-off events.

2. The geometrical configuration of the glacier is similar before each break-off, with an upper crevasse spanning the whole glacier width and a clear thickening of the glacier towards its tongue.

3. The upper crevasse marks a clear distinction between a stable upper part (where Stake 4 is located) and a downstream unstable part (where the other reflectors were located; Fig. 2, Sect. 4.1). A crude estimation of the volume of the unstable part is thus possible.

4. Downstream of this crevasse, surface displacements experience a typical acceleration prior to break-off, whereas upstream of this crevasse, constant velocities are recorded (Stake 4 in Fig. 3).

5. The rupture took place immediately above the ice/bedrock interface, probably within the ice (Fig. 2). However, this observation remains imprecise since no length scale is available. Therefore no definitive conclusions on the fracture location can be drawn from this observation. Note that a similar observation on fracture location was mentioned for the Weisshorn break-off event, probably due to bedrock irregularities (Pralong and Funk, 2006).

6. The whole break-off occurred in two steps; a minor section at the left side of the glacier was released first. 


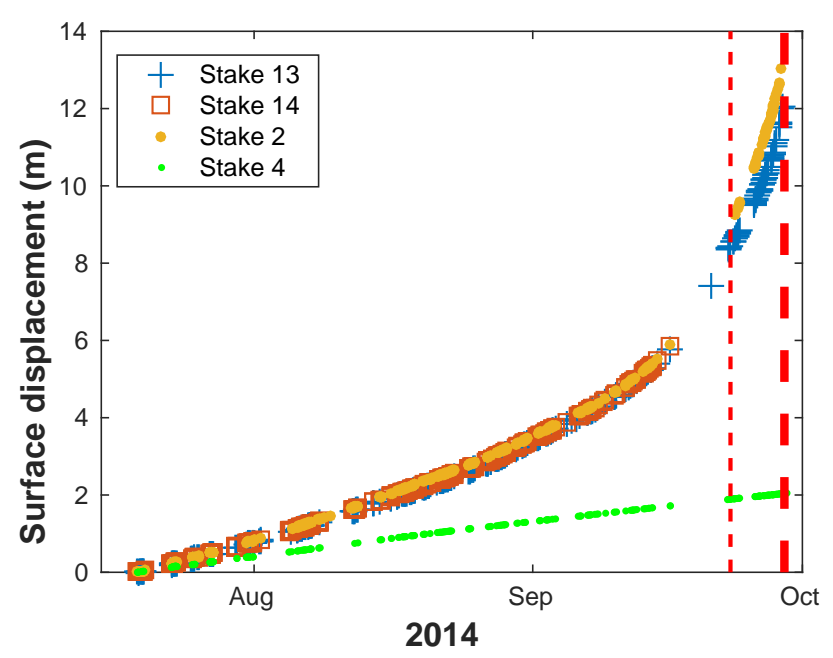

Figure 3. Surface displacements of the four stakes before the breakoff using 19 July 2014 as reference (when stakes 2 and 4 were installed). Vertical red dashed lines indicate the occurrence of the two break-offs, on 23 and 29 September 2014. Interrupted lines indicate a period of bad weather conditions without measurements. Note that Stake 14 was not surveyed after 16 September 2014, i.e., 1 week before the first break-off.

\section{Previous findings on cold glacier break-off}

Based on a retrospective analysis, the main conclusion drawn by Flotron (1977) and Röthlisberger (1981) was that the forecast of a break-off event from a hanging glacier was possible using surface displacements alone. The principle is to fit the characteristic acceleration of the surface motion with a power law behavior of the form:

$s(t)=s_{0}+u_{\mathrm{s}} t-a\left(t_{\mathrm{c}}-t\right)^{\theta}$,

where $s(t)$ is the displacement (in meters) at time $t$ (in days), $s_{0}$ a constant in meters, $u_{\mathrm{S}}$ the constant velocity of the upstream stable part (in $\left.\mathrm{md}^{-1}\right), t_{\mathrm{c}}$ the critical time (in days), $\theta<0$ (without units) and $a$ (in $\mathrm{md}^{-\theta}$ ) the parameters characterizing the acceleration. In this way, the critical time, $t_{\mathrm{c}}$, i.e., the time at which the theoretical displacement becomes infinite, could be evaluated using such an empirical law. Although the break-off event would necessarily occur earlier, this critical time represents the upper limit of the break-off timing. Moreover, an oscillating pattern superimposed on the power law acceleration of the surface displacements was evidenced prior to the 2005 Weisshorn event (Pralong et al., 2005; Faillettaz et al., 2008). This peculiar glacier dynamics was shown to be a log-periodic oscillating process superimposed on this acceleration (for appearance and interpretation see Pralong, 2006 and Faillettaz et al., 2015). The time evolution of the surface displacement measurements can be described with the following equation (after Sornette and Sam- mis, 1995; Pralong et al., 2005):

$s(t)=s_{0}+u_{\mathrm{s}} t-a\left(t_{\mathrm{c}}-t\right)^{\theta}\left[1+C \sin \left(2 \pi \frac{\ln \left(t_{\mathrm{c}}-t\right)}{\ln (\lambda)}+D\right)\right]$,

where $C$ is the relative amplitude (without units), $\lambda$ the logarithmic frequency (in days) and $D$ the phase shift of the log-periodic oscillation (without units).

Thanks to a combined analysis of surface displacement and seismic measurement, Faillettaz et al. (2011a) were able to obtain a coherent quantitative picture of the damage evolution process developing before the 2005 Weisshorn breakoff. They have suggested three regimes in the evolution of the failure process leading to the break-off event:

i. an initial stable phase related to a self-organizing regime, where diffuse damage accumulates within the glacier, with a proliferation of dislocation-like defects;

ii. a transitional phase where the damage process goes on, micro-cracks grow and start merging in a homogeneous way; log-periodic oscillations appear and reveal the hierarchical structure of the fracture process under development;

iii. a catastrophic regime where damage clusters are randomly activated; damage clusters interact and merge with a preferential direction (i.e., preparing the final rupture pattern), in contrast to the previous regime.

\section{Results}

\subsection{Surface displacements and associated velocities}

Figure 3 shows the corrected surface displacements and Fig. 4 the associated derived surface velocities of the four stakes (Fig. 2) prior to the break-off. The associated derived surface velocities are computed by taking the surface displacements (smoothed over five points) interpolated on a regular time step of 1 day. Note that stakes 13 and 14 have more than 2 years of nearly continuous measurements and the position of Stake 13 was surveyed up to the final breakoff event on 29 September. Because of the long distance between the theodolite and the reflectors, a small error in angle measurement has considerable impact on the accuracy of the calculated position. Following Faillettaz et al. (2008), two corrections were applied to the raw data to improve the accuracy. First, the distance was corrected using two reference points located on the rock next to the glacier to compensate the meteorological disturbances of the air temperature, humidity and pressure. Second, assuming that a material point moves along a straight streamline, the reflector position can be determined by using only the measured distance, as each measurement is associated with a unique position on the line. Finally the error of the results was estimated to be less than $1 \mathrm{~cm}$. Note that this constitutes a unique data set, not only 


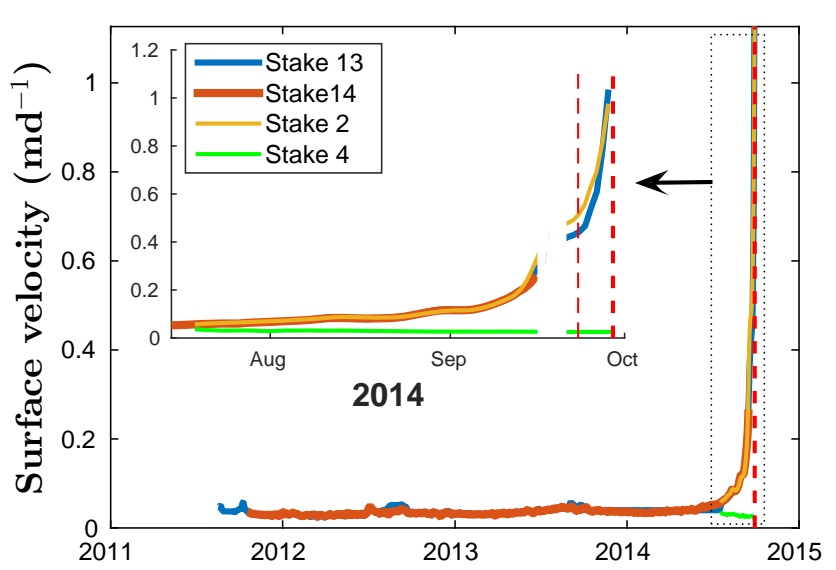

Figure 4. Surface velocities for four stakes since 2012. Inset shows a closer view during the same period as in Fig. 3.

because of the great accuracy and long measurement period, but also due to available surface displacement data up to a few hours prior to the break-off event. Whereas surface velocities at Stake 4 are approximately constant (Fig. 4), the three other stakes show a clear acceleration which is typical for an unstable situation. According to this observation we can expect that the glacier section around stakes 2, 13 and 14 will break off, while the section around Stake 2 will remain stable (Sect. 2.4).

\subsection{Application to forecasting}

Previous findings (Sect. 3) were applied in order to forecast the breaking-off event in real time. As soon as a significant increase in velocity was detected, the same procedure was followed as in Faillettaz et al. (2008). We periodically fitted surface displacements of all stakes to a power law (Eq. 1) and a log-periodic oscillating behavior (Eq. 2). The nonlinear least-squares curve fitting was performed using the Levenberg-Marquardt algorithm. Because the results depend on the initial parameter estimates, especially $t_{\mathrm{c}}$ and $\theta$, we have systematically used different initial values with a prescribed bound and selected the results corresponding to the best root-mean-square error and the degree-of-freedomadjusted coefficient of determination.

Figures 5a and 6a show both power law (Eq. 1) and logperiodic (Eq. 2) fits using the last month of available data, i.e., up to 16 September for Stake 14 and 29 September for Stake 13 . As both fits are barely distinguishable, we have also plotted the residuals to the power law fit in Figs. 5b and 6b and show the associated log-periodic fit (minus the power law fit) as a dashed gray line; Table 1 contains the values of the parameters in Eq. (2), taking $\lambda=2$ days. Note that measurements are available up to the final break-off for three prisms (i.e., stakes 13, 2, 4) and stopped on 16 September for Stake 14, i.e., 8 days before the first break-off.
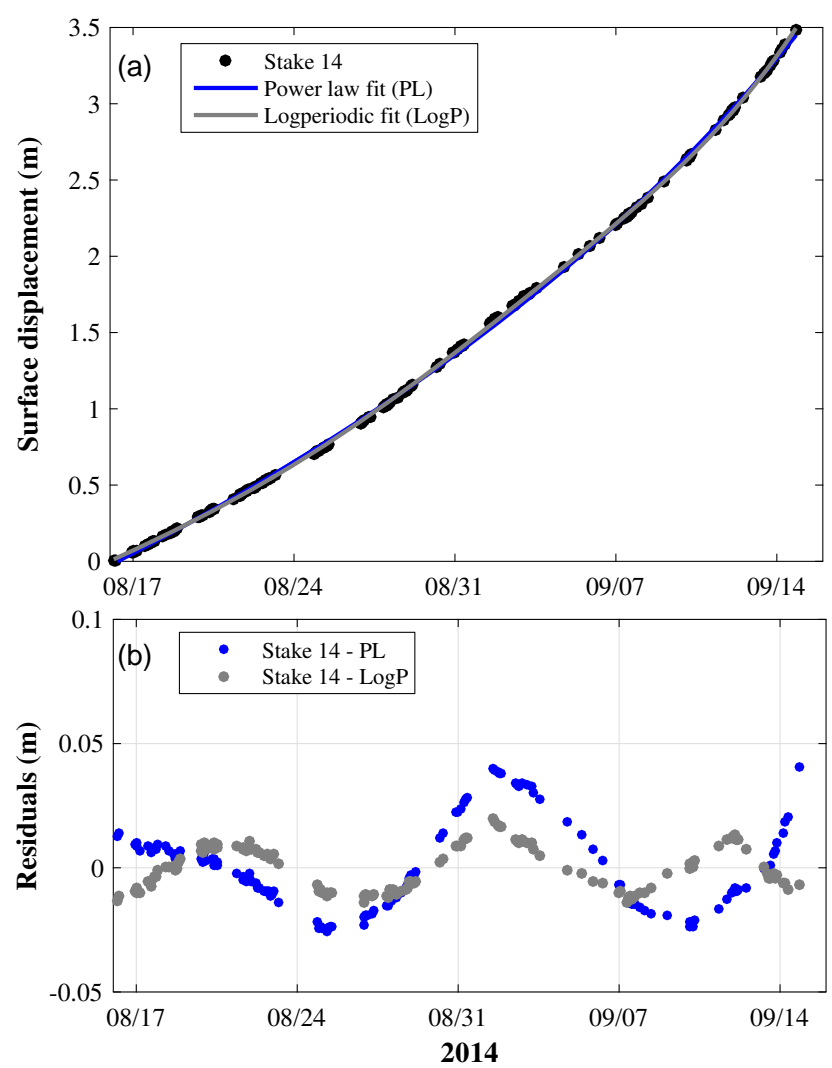

Figure 5. (a) Surface displacements of Stake 14 for the period 16 August-16 September (last measurement of Stake 14) and the associated power law (in blue) and log-periodic fit (in gray). (b) Residuals (in meters) to the power law fit (in blue) and to the log-periodic fit (in gray) for the same period. Values for the parameters are shown in Table 1.

It appears that the power law behavior describes the surface displacements well, with a maximum discrepancy of about $5 \mathrm{~cm}$ for Stake 14 (8 days before break-off), about the same order of magnitude as the one observed during the 2005 Weisshorn event (Fig. 5). However, residuals indicate an oscillating pattern. When using the log-periodic function (Eq. 2), the agreement between measured and fitted values (dashed gray line) becomes better, with an accuracy of the order of magnitude of the measurement accuracy (less than $1 \mathrm{~cm}$ ). Results show that the critical time can be expected around the 3rd October for both stakes, which is fairly close to the observed break-off. Note that such an approach can be used to investigate how far in advance a reliable time forecast is possible (see Sect. 5.4).

However, even if Stake 14 is located on a section that had broken off earlier, no differences could be detected. Our approach is not able to detect whether the break-off will occur all at once or as successive small events.

Now when considering the entire data set for Stake 13 (where measurements could be recorded up to the break-off) using the same method, it appears that the amplitudes of the 
Table 1. Values of the estimated coefficients of Eq. (2) with $\lambda=2$ days and the root-mean-square error (RMSE) of the fit, the first two columns corresponding to the parameters of the fit used in Fig. 5 for the period 16 August-16 September, the last two columns corresponding to the parameters of the fit used in Fig. 6 for the period 30 August-30 September. $t_{\mathrm{c}}$ is given in days after the first day of the investigated period.

\begin{tabular}{|c|c|c|c|c|c|}
\hline \multirow[t]{2}{*}{ Parameter } & \multirow[t]{2}{*}{ Units } & Stake 13 & Stake 14 & Stake 13 & Stake 2 \\
\hline & & \multicolumn{2}{|c|}{ (16 August-16 September) } & \multicolumn{2}{|c|}{ (29 August-29 September) } \\
\hline \multirow[t]{2}{*}{$t_{\mathrm{c}}$} & days & $48.02 \pm 5.13$ & $48.11 \pm 5.6$ & $41.93 \pm 0.91$ & $41.80 \pm 2.18$ \\
\hline & date & 3 Oct 2014 & 3 Oct 2014 & 10 Oct 2014 & 10 Oct-2014 \\
\hline$\theta$ & - & -0.24 & -0.25 & -0.99 & -0.98 \\
\hline$s_{0}$ & $\mathrm{~m}$ & $-1.47 \times 10^{4}$ & $-1.47 \times 10^{4}$ & $-2.03 \times 10^{4}$ & $-1.48 \times 10^{4}$ \\
\hline$u_{s}$ & $\mathrm{md}^{-1}$ & $2.00 \times 10^{-2}$ & $2.00 \times 10^{-2}$ & $2.99 \times 10^{-2}$ & $2.27 \times 10^{-2}$ \\
\hline$a$ & $\mathrm{md}^{-\theta}$ & 27.88 & 27.72 & 141.73 & 164.42 \\
\hline$C$ & - & $2.9 \times 10^{-3}$ & $2.3 \times 10^{-3}$ & $3.0 \times 10^{-2}$ & $2.0 \times 10^{-2}$ \\
\hline$D$ & - & 2.25 & 1.97 & 6.13 & 0.06 \\
\hline RMSE & $\mathrm{md}^{-1} \mathrm{~m}^{-1}$ & $8.7 \times 10^{-3}$ & $7.9 \times 10^{-3}$ & $3.05 \times 10^{-2}$ & $2.51 \times 10^{-2}$ \\
\hline
\end{tabular}

oscillations superimposed on the power law acceleration become even larger close to the break-off - they reach values up to $30-40 \mathrm{~cm}$ (Fig. 6). Such a broad oscillating pattern has never been observed before, confirming that the jerky motion of the glacier (with oscillating nature) might have a physical origin (see Sect. 5.2).

\section{Discussion}

\subsection{Influence of data accuracy on the final result}

To assess how the data accuracy influences the time forecast of the break-off, and to analyze how the obtained critical time depends on our fitting method, we artificially added two uniformly distributed instances of random noise (between -1 and $1 \mathrm{~cm}$ and between -5 and $5 \mathrm{~cm}$ ) to our data set. To ensure good statistical representation, this procedure was performed 100 times on Stake 14 up to 16 September 2014 and on Stake 13 up to the final break-off (29 September 2014). Results are shown in Table 2 where errors (i.e., $95 \%$ confidence interval) associated to the additional noise (in bracket) and to the fitting procedure are also reported. These results show that (i) data accuracy does not influence the value of the forecast (less than 0.5 day); (ii) data accuracy directly influences the confidence of the fit (decreasing data accuracy affects the confidence interval of the fit drastically); (iii) the confidence interval of the fit is reduced if data (even with low accuracy) can be collected up to the break-off. Therefore, the data accuracy directly determines how far in advance a satisfying forecast can be achieved (see Sect. 5.4).

\subsection{Appearance of log-periodic behavior}

The origin of the log-periodic oscillating behavior is likely due to a discrete scale invariance (DSI). DSI is a weaker kind of scale invariance according to which the system obeys

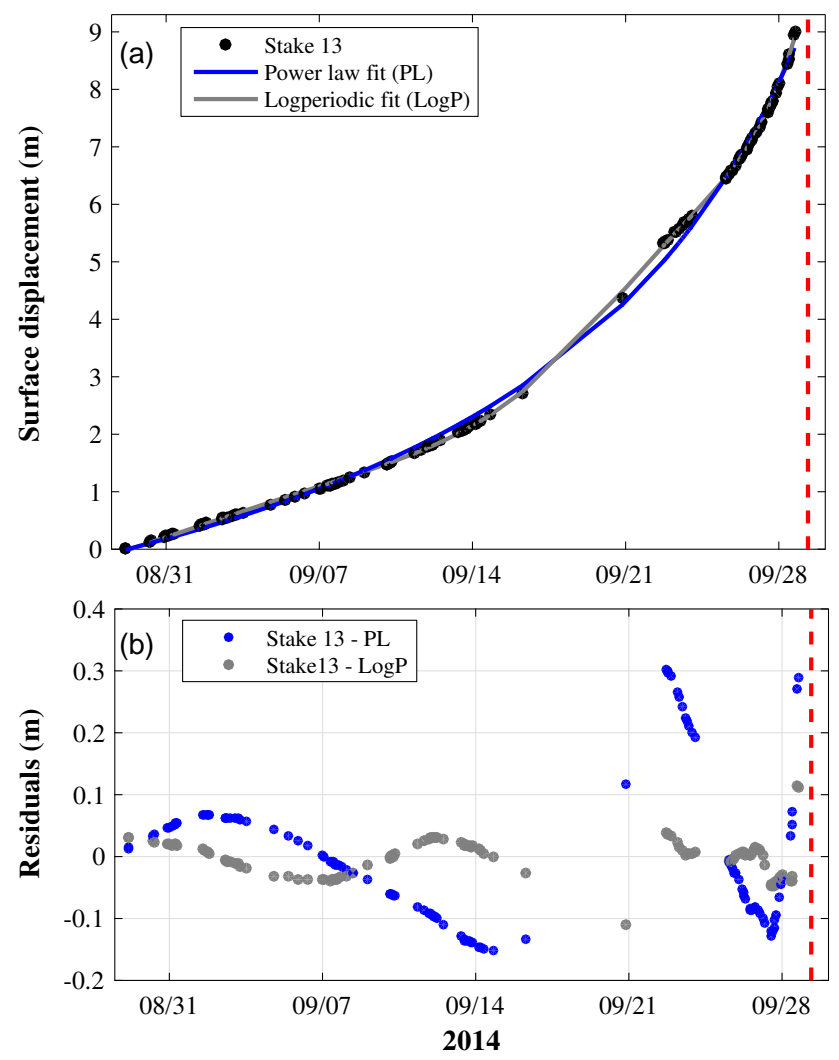

Figure 6. (a) Surface displacement of Stake 13 for the period 29 August 2014-29 September 2014 and associated power law (in blue) and log-periodic (in gray) fits. (b) Residuals (in meters) to the power law fit (blue) and to the log-periodic fit (in gray) for the same period. Values of the parameters are shown in Table 1.

scale invariance only at a specific scaling factor (Sornette and Sammis, 1995; Sornette, 1998; Zhou and Sornette, 2002a; Sornette, 2006). Whereas the hallmark of continuous scale 
Table 2. Critical time $t_{\mathrm{c}}$ evaluated using our initial data set and with additional uniformly distributed random noise of \pm 1 and $\pm 5 \mathrm{~cm}$ (over 100 realizations). The errors (i.e., $95 \%$ confidence interval) resulting for the additional noise (values italicized in brackets) and from the fit are also reported.

\begin{tabular}{lccc}
\hline Stake & Initial data & $\pm 1 \mathrm{~cm}$ noise & $\pm 5 \mathrm{~cm}$ noise \\
\hline 13 & $t_{\mathrm{c}}=41.93 \pm 0.91$ day & $t_{\mathrm{c}}=41.90[ \pm 0.038] \pm 0.9$ day & $t_{\mathrm{c}}=41.86[ \pm 0.041] \pm 1.2$ day \\
14 & $t_{\mathrm{c}}=48.11 \pm 5.6$ days & $t_{\mathrm{c}}=48.55[ \pm 0.56] \pm 8.9$ days & $t_{\mathrm{c}}=49.33[ \pm 0.55] \pm 34.2$ days \\
\hline
\end{tabular}

invariance is the existence of power law, the signature of DSI is the presence of power laws with complex exponents which manifests itself in data by log-periodic corrections to scaling. Several mechanisms may lead to this partial breaking of the continuous symmetry. Thanks to a combined analysis of surface displacements and seismic measurements, Faillettaz et al. (2011a) suggest that it results from the dynamic interactions between newly developed micro-cracks, as shown by Huang et al. (1997) and Sahimi and Arbabi (1996).

To identify the $\log$ frequency, we analyzed the data in the same way as Faillettaz et al. (2008) with a Lomb periodogram analysis (Press, 1996; Zhou and Sornette, 2002b), which is designed to analyze nonuniformly sampled time series. This method enables us to determine $f_{\text {Lomb }}$ as a function of $\cos \left(2 \pi f_{\text {Lomb }} t\right)$. The parameter $\lambda$ in Eq. (2) can then be evaluated easily as $\lambda=e^{1 / f_{\text {Lomb }}}$. Unfortunately, the critical time, $t_{\mathrm{c}}$, has to be known to perform this analysis; i.e., this analysis can only be performed a posteriori. Figure 7 shows a Lomb periodogram analysis for the three stakes, accounting for their displacements before the first break-off using the final break-off as the critical time. A common peak is clearly visible at $f_{\text {Lomb }}=1.45 \pm 0.1$ day $\leftrightarrow \lambda \sim 2 \pm 0.1$ day, thus confirming that the oscillating behavior is not a measurement artifact but has physical origins, such as the merging of newly developed micro-cracks. Note that this $\lambda$ value is not only compatible with previous findings on such types of breakoff (Faillettaz et al., 2008), but also with other phenomena such as growth processes (Sornette et al., 1996), earthquakes (Sornette and Sammis, 1995) or financial crashes (Sornette and Johansen, 2001).

Interestingly, when analyzing only the data collected after the first break-off, i.e., for stakes 2 and 13, another strong $\log$ frequency appears at $\lambda_{2} \sim 4.45 \pm 1$ day in addition to the previous peak at $\lambda_{1} \sim 2$ days (Fig. 8). A similar peak was also observed when analyzing the Weisshorn 2005 break-off. The reason for both the appearance and the value of such a subharmonic peak at $\lambda_{2}$ after the first break-off is not clear.

However, Sornette et al. (1996) and Huang et al. (1997) suggested that, for the growth of the population of cracks oriented in one direction, such subharmonic frequencies appear naturally and are arbitrary powers, $\lambda^{n}$, of the preferred scaling ratio, $\lambda$. Such a mechanism could thus explain the peak at $\lambda_{2} \sim \lambda_{1}^{2}$. This peak clearly occurred after the first break-off, suggesting that this event is at the origin of its appearance. The vibrations generated by this ice mass release

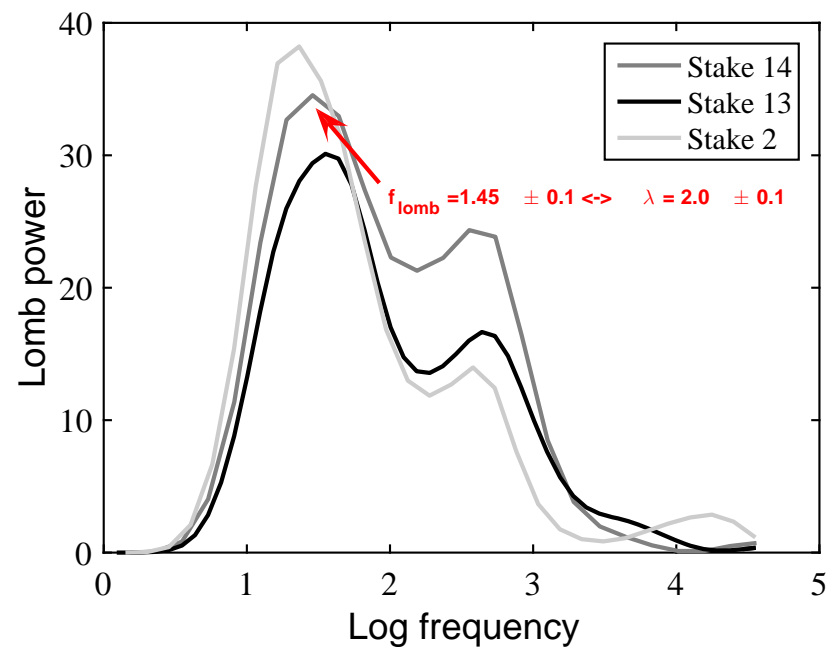

Figure 7. Lomb periodogram for stakes 13,14 and 2 before the first break-off event as well as the corresponding log frequencies $(\lambda)$ of the peaks.

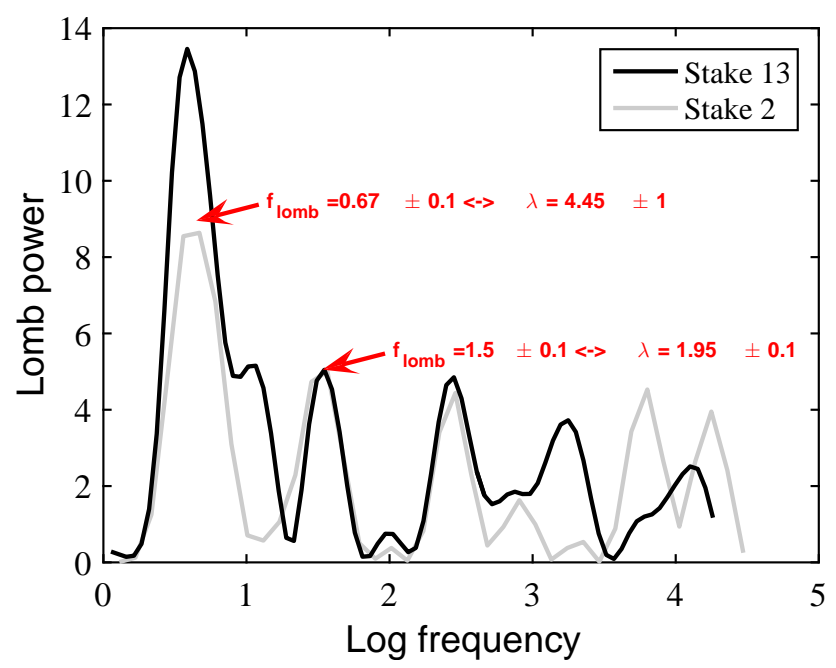

Figure 8. Lomb periodogram for stakes 13 and 2 after the first break-off event as well as the corresponding log frequencies $(\lambda)$ of the peaks.

and its consecutive avalanche might generate a sudden additional increase in the internal damage of the remaining section (where stakes 13 and 4 are located). This supplementary 
external load perturbs the hierarchical self-organization of the micro-cracks, presumably promoting other subharmonic peaks.

Another possible explanation for the appearance of logperiodic behavior with different harmonic peaks after the first break-off could result from a perturbation in the hierarchy of cracking; the pure log periodicity assumes a single discrete hierarchy. It seems that the first large rupture may lead to a nonlinear distortion of the subsequent development of the hierarchy of cracking, with a drift in the log frequency. This kind of phenomenon was modeled using a nonlinear secondorder (or third-order) Landau expansion of the Log-Periodic Power Law (LPPL) formalism for application to financial markets (Johansen and Sornette, 1999; Zhou and Sornette, 2004).

\subsection{Accurate determination of break-off occurrence}

Critical time, $t_{\mathrm{c}}$, given by power law or log-periodic fit indicates when surface displacements become theoretically infinite. However, the real break-off event is expected before $t_{\mathrm{c}}$. When fitting the surface displacements with both power law and log-periodic behavior in real time, it is not only possible to assess the critical time, but also the time at which the derived velocities are expected to reach a given threshold. Fitting and estimating the time at which the velocity reaches a given threshold provides a more accurate way to predict the real break-off event. We developed software based on this idea by fitting the measurements with both power law and log-periodic behavior in real time, and thus providing an estimate of the break-off time. Note that the proposed method for determining the break-off time depends on the choice of the threshold velocity; choosing a small threshold value is conservative in terms of security as a smaller velocity is reached first for the same fit. Moreover using a range of threshold velocity for estimating the break-off time allows a time interval to be provided in which the break-off event is likely to occur.

According to our knowledge, it is not possible to know in advance the velocity at which break-off will occur. However, from previous events (Weisshorn 1973 and 2005 event, Flotron, 1977; Röthlisberger, 1981; Faillettaz et al., 2008), it seems that break-off occurs between 0.5 and $1.2 \mathrm{md}^{-1}$; but this is based on a restricted number of events.

Arbitrarily taking threshold surface velocities of 0.5 and $1 \mathrm{~m} \mathrm{~d}^{-1}$ for possible lower and upper velocity limits at which break-off could occur, our analysis (using Eq. 2) performed every day from 12 August to 16 September suggested that a break-off event was likely to occur between 23 September $\left(v_{\text {th }}=0.5 \mathrm{~m} \mathrm{~d}^{-1}\right)$ and 29 September $\left(v_{\text {th }}=1 \mathrm{md}^{-1}\right)$.

Following this analysis, an alert was immediately sent to the authorities, leading them to close the endangered area 1 week before the event. Note again that the definition of the velocity threshold has an influence on the prediction itself, as we saw nearly 1 week is needed for the glacier to accelerate from 0.5 to $1 \mathrm{md}^{-1}$. The precise prediction would also not only be based on a correct fit of the surface displacement data, but also on a guess of this parameter. It is not yet clear which value has to be considered according to the results from the events analyzed so far; but we suggest choosing $0.40 \mathrm{md}^{-1}$ as a conservative threshold to define a safe break-off danger time interval.

\subsection{How far in advance are time forecasts possible?}

Surface displacements were analyzed retrospectively based on the last month of data for each stake, and the critical time as well as the time at which the fitted velocity reached $0.5 \mathrm{md}^{-1}\left(v_{50}\right)$ and $1 \mathrm{md}^{-1}\left(v_{100}\right)$ were plotted as a function of the time (Fig. 9). Associated errors (right panels) account for the fitting procedure.

This retrospective analysis shows that the prediction is correct after 12 September, i.e., 11 and 17 days before the breakoff, with a confidence interval becoming less than 10 days with a log-periodic fit. This analysis points out the great prediction potential - and early-warning perspective - of this method, as the time of the break-off could be forecasted almost 2 weeks in advance. Note that the log-periodic fits become less accurate after the first break-off for Stake 13 (Fig. 9b). This might be related to the occurrence of the first break-off that had possibly changed the hierarchical organization of the internal damage (see Sect. 5.2). However, note that the time at which a velocity of $1 \mathrm{md}^{-1}\left(v_{100}\right)$ is expected remains unaffected, still pointing at 29 September.

\subsection{Overall recommendations}

According to the knowledge gained from the different studies on Weisshorn, Mönch and Grandes Jorasses glaciers, accurate data are required to forecast an impeding break-off event. As the amplitudes of the log-periodic oscillations are increasing towards the break-off (from $5 \mathrm{~cm} 1$ week before the break-off to $40 \mathrm{~cm}$ at the break-off), the confidence of the time forecast strongly depends on the precision of the surveying data. To ensure a satisfactory forecast about 1 week in advance, a surveying accuracy better than half of the expected log-periodic amplitudes, i.e., $2.5 \mathrm{~cm}$, is required. In this study, an accuracy of $1 \mathrm{~cm}$ was achieved with an automatic total station (Leica theodolite TM1800 combined with the DI3000S Distometer). The sampling rate needs to be adapted to the oscillating pattern in order to enable its detection. Moreover, in such rapidly changing meteorology where clouds can momentarily hinder measurements, several measurements need to be performed each day. A sampling rate of $2 \mathrm{~h}$ was chosen in this study, thus ensuring several opportunities to obtain data every day. This technique can be performed in near-real time and several measurements can be performed every day with a sufficient accuracy. Note that GPS measurements would be a valuable alternative but this technique requires a long acquisition time and additional processing to achieve required accuracy. Although indepen- 
(a)

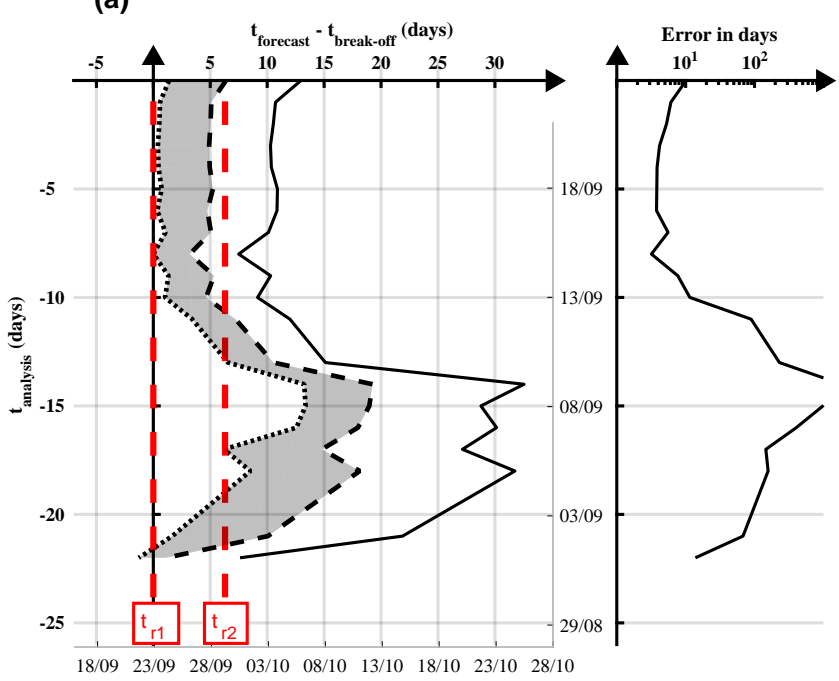

(b)

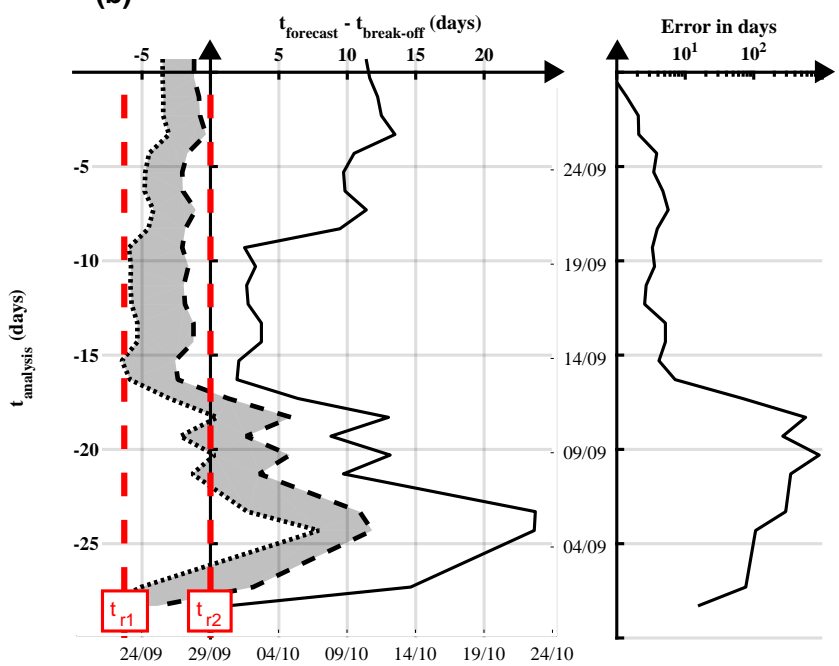

Figure 9. Evaluated critical time, $t_{\mathrm{c}}$, for log-periodic fit (black thick line) for Stake 14 (a, left) and Stake 13 (b, left) as a function of the time of analysis using the last month of data ( $\left.t_{\text {analysis }}\right)$. Shaded area represents the most likely break-off occurrence. This area is delimited by interrupted lines, indicating the time at which the estimated derived velocity log-periodic fit reaches $0.5 \mathrm{md}^{-1}$ (dashed lines, $v_{50}$ ) and $1 \mathrm{md}^{-1}$ (dot-dash line, $v_{100}$ ). The break-off (corresponding to 0 on horizontal axis) occurred on (a) 23 September $\left(t_{\mathrm{r} 1}\right)$ and (b) 29 September $\left(t_{\mathrm{r} 2}\right)$. Vertical red lines indicate the observed break-offs $\left(t_{\mathrm{r} 1}\right.$ and $\left.t_{\mathrm{r} 2}\right)$. Right: error in days on critical time fitted with log-periodic estimated from the $95 \%$ confidence interval as a function of the time prior to the break-off event ( $\left.t_{\text {analysis }}\right)$. Errors on $v_{50}$ and $v_{100}$ are similar to the errors on critical time, as they are directly derived from these fits. dent of weather conditions, the power supply and data transmission are problems to be solved. This procedure based on power law/log-periodic oscillations regression requires at least two measurement points on the potentially unstable part of the glacier, so that the time evolution of surface motion at different points can be compared. It also ensures that the results are not affected by stake/prism stability issues.

An alternative surveying technique is terrestrial InSAR (interferometric synthetic aperture radar). The advantage of this technique is that no installation on the glacier (potentially dangerous) is required. However the data accuracy which can be expected with this monitoring system is not completely clear yet (Preiswerk et al., 2016).

\section{Conclusions}

Grandes Jorasses glacier broke off twice, on 23 and 29 September 2014. In 2008, as it was suspected that a large part of this glacier was becoming unstable, a monitoring program was initiated. At the time of the break-off, four stakes covering a large part of the glacier enabled surface displacement measurements up to the time of the break-off. By regularly analyzing the data set, it was possible to forecast the event 10 days in advance. Subsequently, the local authorities closed the endangered area up to the final rupture.

It was possible to confirm for an impeding ice fall that a time series of surface displacements exhibits strong logperiodic oscillations superimposed on a global power law acceleration, as first discovered for the Weisshorn event (Faillettaz et al., 2015). In the immediate vicinity of the break-off, such oscillations reached an amplitude of more than $40 \mathrm{~cm}$, almost 1 order of magnitude larger than revealed in previous findings. By fitting our recorded surface displacements, the critical time, i.e., the time at which surface displacement become infinite, can be determined. Using this critical time value as an upper bound, a good time forecast could be achieved.

The inferred surface velocities immediately prior to the two events were $0.5 \mathrm{md}^{-1}$ for 23 September and $1.2 \mathrm{md}^{-1}$ for 29 September, in the same range as for the Weisshorn event, suggesting that break-off of a cold hanging glacier can occur as soon as surface velocities reach $0.5 \mathrm{md}^{-1}$. We showed that evaluating the time at which extrapolated velocities (based on the log-periodic fit) reach a prescribed threshold $\left(0.5\right.$ and $\left.1 \mathrm{~m} \mathrm{~d}^{-1}\right)$ provides a better forecast. However, in the present case, surface velocity increased from 0.5 to $1 \mathrm{md}^{-1}$ in the order of 1 week. In practice, we suggest using a critical velocity of $v=0.4 \mathrm{md}^{-1}$ to determine the period of highly likely break-off occurrence. A retrospective analysis based on this method showed that an accurate prediction of the phenomenon can be achieved 2 weeks before its occurrence using the last month of surface displacement data and 0.5 and $1 \mathrm{md}^{-1}$ as velocity thresholds. Although enabling a crude estimation of the total unstable ice volume, this point- 
based surveying procedure is not appropriate to determine whether the unstable ice mass will fall down in one event or disaggregate and give rise to several smaller events, as no differences in the evolution of surface displacements were detected. This has consequences for the risk evaluation, as the resulting ice avalanche (and also the chain of processes resulting from its release) depends on the falling ice volume. To conclude, our results suggest that the presented monitoring and data processing techniques exploiting log-periodic oscillating behavior can be applied in real time to forecast a break-off event on any cold unstable hanging glacier.

Acknowledgements. The authors thank Susan Braun-Clarke for proofreading the English. The authors thanks also J. Bassis and two anonymous reviewers for their useful comments that significantly improved the manuscript.

Edited by: O. Gagliardini

\section{References}

Christen, M., Kowalski, J., and Bartelt, P.: RAMMS: Numerical simulation of dense snow avalanches in three-dimensional terrain, Cold Reg. Sci. Technol., 63, 1-14, 2010.

Faillettaz, J., Pralong, A., Funk, M., and Deichmann, N.: Evidence of log-periodic oscillations and increasing icequake activity during the breaking-off of large ice masses, J. Glaciol., 54, 725-737, doi:10.3189/002214308786570845, 2008.

Faillettaz, J., Funk, M., and Sornette, D.: Icequakes coupled with surface displacements for predicting glacier break-off, J. Glaciol., 57, 453-460, doi:10.3189/002214311796905668, 2011a.

Faillettaz, J., Sornette, D., and Funk, M.: Numerical modeling of a gravity-driven instability of a cold hanging glacier: reanalysis of the 1895 break-off of Altelsgletscher, Switzerland, J. Glaciol., 57, 817-831, doi:10.3189/002214311798043852, 2011b.

Faillettaz, J., Funk, M., and Sornette, D.: Instabilities on Alpine temperate glaciers: new insights arising from the numerical modelling of Allalingletscher (Valais, Switzerland), Nat. Hazards Earth Syst. Sci., 12, 2977-2991, doi:10.5194/nhess-12-29772012, 2012.

Faillettaz, J., Funk, M., and Vincent, C.: Avalanching glacier instabilities: review on processes and early warning perspectives, Rev. Geophys., 53, 203-224, doi:10.1002/2014RG000466, 2015.

Flotron, A.: Movement studies on hanging glaciers in relation with an ice avalanche, J. Glaciol., 19, 671-672, 1977.

Huang, Y., Ouillon, G., Saleur, H., and Sornette, D.: Spontaneous generation of discrete scale invariance in growth models, Phys. Rev. E, 55, 6433-6447, doi:10.1103/PhysRevE.55.6433, 1997.

Johansen, A. and Sornette, D.: Financial "anti-bubbles": Logperiodicity in gold and Nikkei collapses, Int. J. Mod. Phys. C, 10, 563-575, doi:10.1142/S0129183199000437, 1999.

Margreth, S., Faillettaz, J., Funk, M., Vagliasindi, M., Diotri, F., and Broccolato, M.: Safety concept for hazards caused by ice avalanches from Whymper hanging glacier in the Mont-Blanc massif, Cold Reg. Sci. Technol., 69, 194-201, doi:10.1016/j.coldregions.2011.03.006, 2011.
Pralong, A.: Oscillations in critical shearing, application to fractures in glaciers, Nonlin. Processes Geophys., 13, 681-693, doi:10.5194/npg-13-681-2006, 2006.

Pralong, A. and Funk, M.: On the instability of avalanching glaciers, J. Glaciol., 52, 31-48, doi:10.3189/172756506781828980, 2006.

Pralong, A., Birrer, C., Stahel, W. A., and Funk, M.: On the predictability of ice avalanches, Nonlin. Processes Geophys., 12, 849-861, doi:10.5194/npg-12-849-2005, 2005.

Preiswerk, L., Wlater, F., Anandakrishnan, S., Barfucci, G., Beutel, J., Burkett, P., Dalban, P., Funk, M., Limpach, P., Marchetti, E., Meier, L., and Neyer, F.: Monitoring unstable parts in the ice-coeverd Weissmies northwest face, in: Proceedings of INTRAPRAEVENT 2016, 30 May-2 June 2016, Lucerne, Switzerland, in press, 2016.

Press, W.: Numerical Recipes in Fortran 90: Volume 2 of Fortran Numerical Recipes: The Art of Parallel Scientific Computing, Fortran numerical recipes, Cambridge University Press, available at: http://books.google.ch/books?id=SPEi4mCfhacC, last access: May 2016, 1996.

Röthlisberger, H.: Eislawinen und Ausbrüche von Gletscherseen, in: Gletscher und Klima - glaciers et climat, Jahrbuch der Schweizerischen Naturforschenden Gesellschaft, wissenschaftlicher Teil 1978, edited by: Kasser, P., Birkhäuser Verlag Basel, Boston, Stuttgart, 170-212, 1981.

Sahimi, M. and Arbabi, S.: Scaling Laws for Fracture of Heterogeneous Materials and Rock, Phys. Rev. Lett., 77, 3689-3692, doi:10.1103/PhysRevLett.77.3689, 1996.

Sornette, D.: Discrete-scale invariance and complex dimensions, Phys. Rep., 297, 239-270, doi:10.1016/S0370-1573(97)000768, 1998.

Sornette, D.: Critical Phenomena in Natural Sciences: Chaos, Fractals, Selforganization and Disorder: Concepts and Tools, Springer Series in Synergetics, Springer, available at: http:// books.google.com.au/books?id=uYHQHV2hCpwC, last access: May 2016, 2006.

Sornette, D. and Johansen, A.: Significance of log-periodic precursors to financial crashes, Quant. Financ., 1, 452-471, doi:10.1088/1469-7688/1/4/305, 2001.

Sornette, D. and Sammis, C. G.: Complex Critical Exponents from Renormalization Group Theory of Earthquakes: Implications for Earthquake Predictions, J. Phys. I, 5, 607-619, doi:10.1051/jp1:1995154, 1995.

Sornette, D., Johansen, A., Arneodo, A., Muzy, J. F., and Saleur, H.: Complex Fractal Dimensions Describe the Hierarchical Structure of Diffusion-Limited-Aggregate Clusters, Phys. Rev. Lett., 76, 251-254, doi:10.1103/PhysRevLett.76.251, 1996.

Zhou, W.-X. and Sornette, D.: Generalized q analysis of logperiodicity: Applications to critical ruptures, Phys. Rev. E, 66, 046111, doi:10.1103/PhysRevE.66.046111, 2002a.

Zhou, W.-X. and Sornette, D.: Evidence of intermittent cascades from discrete hierarchical dissipation in turbulence, Physica D, 165, 94-125, doi:10.1016/S0167-2789(02)00390-1, 2002b.

Zhou, W.-X. and Sornette, D.: Antibubble and prediction of China's stock market and real-estate, Physica A, 337, 243-268, doi:10.1016/j.physa.2004.01.051, 2004. 